\title{
THE CRAB PULSAR AS A COMPONENT OF A \\ BINARY SYSTEM
}

\author{
A. I. TSYGAN
}

A. F. Ioffe Physical Technical Institute, Academy of Sciences, Leningrad, U.S.S.R.

\begin{abstract}
This note discusses the properties of the Crab pulsar assuming that it is moving in the gravitational field of the second component.
\end{abstract}

The concept that the pulsar NP 0532 in the Crab Nebula is a component of a binary system is considered. If this is the case, it is most probable that the second component of the system is the so-called south-preceding star (Shklovsky, 1968). In the framework of the binary model the observed slow-down index of the neutron star $n=$ $=2.16 \pm 0.1$ (Richards et al., 1970), $n=2.4 \pm 0.2$ (Boynton et al., 1972) might be brought in correspondence to the slow-down index $n_{0}=3.6$ as obtained from the data on the pulsar age. Ruderman (1972) obtained an average slow-down index $n_{0}=3.4$ for all pulsars. Let us consider the pulsar to move in the gravitational field of the second component of the binary system. The pulse frequency $\omega$ in the reference system of the observer is connected with the pulse frequency $\omega_{0}$ in the proper reference system of the pulsar by the relation:

$$
\omega=\omega_{0}\left(1+\frac{v}{c} \cos \alpha\right)
$$

where $v$ is the linear velocity of the orbital motion.

From (1) one obtains:

$$
n \approx n_{0}+\omega^{2} \ddot{v}(\dot{\omega})^{-2} c^{-1} \cos \alpha .
$$

$n_{0}=\omega_{0} \ddot{\omega}_{0}\left(\dot{\omega}_{0}\right)^{-2}$ is the true slow-down index determined by physical processes near the neutron star $\left(n_{0}=3.6\right)$ and $n=\omega \ddot{\omega}(\dot{\omega})^{-2}$ is the observed index. Relation (2) is obtained on the assumption that the characteristic time of the orbital velocity change $v / \dot{v}$ is less than the characteristic time for changes in the pulse frequency. If we take $\cos \alpha=$ const., then: $\ddot{v} \cos \alpha=\left(n-n_{0}\right)(\dot{\omega})^{2} c \omega^{-2} \approx-5.2 \times 10^{-12} \mathrm{~cm} \mathrm{~s}^{-3}$. If the pulsar orbit has a large eccentricity then:

$$
\begin{aligned}
\ddot{v} & =\frac{M_{2}}{M_{2}+M_{4}} 2\left[G\left(M_{2}+M_{4}\right)\right]^{1 / 3}\left(\frac{T}{2 \pi}\right)^{-7 / 3} f(\xi) \\
f(\xi) & =\frac{\sin \xi}{(1-e \cos \xi)^{4}} ; \quad 0 \leqslant \xi<2 \pi \\
T & =2 \pi a^{3 / 2}\left[G\left(M_{2}+M_{4}\right)\right]^{-1 / 2} ; \quad t=\frac{T}{2 \pi}(\xi-e \sin \xi) .
\end{aligned}
$$


where $M_{4}$ is the mass of the neutron star, $M_{2}$ the mass of the second component of the binary system, $a$ the semi-major axis of the orbit, $e$ the eccentricity, and $T$ the period of orbital motion. For $M_{2}=1.8 M_{\odot}, M_{4}=0.6 M_{\odot}, e \rightarrow 1$, and $\ddot{v} \approx 7 \times 10^{-12} \mathrm{~cm} \mathrm{~s}^{-3}$, one gets $T=2.8 \times 10^{9} \times \mathrm{f}^{3} / 7^{7} \mathrm{~s}$. One must find the point of the orbit where the pulsar is situated at the moment of observation. It is obvious that the relation $m T+t=\tau$ must be fulfilled if the distance between the components of the binary system was at a minimum at the moment of the pulsar creation. Here $m$ is the total number of orbital revolutions completed by the pulsar since the moment of the pulsar creation and $\tau$ is the pulsar age. The slow-down index $n$ is known from observations to remain approximately constant at least over two years (Boynton et al., 1972) which gives the limit on the value of $\dddot{v}$. Hence the following estimations, $\mathrm{f} \sim(1-100), T \sim(100-600) \mathrm{yr}$, and $a \sim(4-15) \times 10^{14} \mathrm{~cm}$ are obtained, where $a(1-e) \sim 10^{12} \mathrm{~cm}$, the angular scale of the binary system being of the order of 0 ."01. From the data on the value of $\dot{n}$, one can get a more precise value of the period $T$ and the pulsar orbital position.

Hyperbolic trajectories are inconsistent with the observed parameters of the Crab pulsar. If a neutron star (or a 'black hole') appears as a component of a binary system (either closely bound or not), it is reasonable to believe that before the supernova explosion the system was a close binary (Shklovsky, 1968) (the distance $\sim 10^{12} \mathrm{~cm}$ ). If a neutron star (or a 'black hole') has an orbit with a large eccentricity, in the periastron position, the distance between the stars can be so small that the accretion on the neutron star can be effective. This can be observed as a sudden appearance of an X-ray source with its subsequent disappearance (see Cen XR-4 (Conner et al., 1969) for example). The intensity and spectrum of such a source are the same as those of an ordinary close binary system (e.g., Her X-1, Cyg X-1).

\section{References}

Boynton, P. E., Groth, E. J., Hutchinson, D. P., Nanos, G. P., Partridge, R. B., Jr. and Wilkinson, D. T.: 1972, Astrophys. J. 175, 217.

Conner, J. P., Evans, W. D. and Belian, R. D.: 1969, Astrophys. J. 157, L157.

Richards, D. W., Pettengill, G. H., Counselman, C.C., III, and Rankin, J. M.: 1970, Astrophys. J. 160, L1.

Ruderman, M.: 1972, Ann. Rev. Astron. Astrophys. 10, 427.

Shklovsky, I. S.: 1968, Supernovae, Wiley-Interscience, London, chap. III.

\section{DISCUSSION}

N. I. Shakura: In order to obtain an orbit with high eccentricity an exactly fixed amount of matter must be ejected in the symmetrical explosion. If the quantity of matter is a little more, the system will disintegrate.

A. I. Tsygan: First, the explosion may be asymmetrical. Second, we might have in this case the realization of an only chance for the orbit to be highly eccentric.

$N$. G. Bochkarev: What has the second star of the Crab Nebula got to do with the given picture?

A. I. Tsygan: The northern star, which is $4^{\prime \prime}$ from the south one has nothing to do with the given material and it is just projected on the nebula. The south star consists of an ordinary star emitting the main quantity of optical radiation observed from the south star and the pulsar which is situated at a distance of 0.01 from the star. 